\title{
Age related somatic mitochondrial DNA deletions in bone
}

\author{
S S Papiha, H Rathod, I Briceno, J Pooley, H K Datta
}

\begin{abstract}
Background-It has been suggested that the accumulation of damage to mitochondrial DNA is a major cause of age related, degenerative disease. Aging is known to cause bone loss leading to a fall in bone mineral density and disruption of bone microarchitecture. However, despite the evidence of age related bone loss, no attempt has been made to detect specific deletions of mitochondrial DNA in the bone of aged individuals.

Aims-To detect bone specific, age related deletions in mitochondrial DNA.

Method-Blood leucocytes and bone biopsies from patients who had undergone orthopaedic surgery were used as a source of mitochondrial DNA and screened for deletions using the polymerase chain reaction.

Results-Although no deletions were detected in the blood mitochondrial DNA, specific deletions in bone mitochondrial DNA were found in three of five elderly subjects.

Conclusion-The findings of this study suggest that there could be a link between mitochondrial DNA deletions and free radical induced apoptosis of bone cells in the development of age related bone loss. (F Clin Pathol 1998;51:117-120)
\end{abstract}

Keywords: mitochondrial deletions; bone; oxidative stress

Department of Orthopaedics and Traumatic Surgery,

The Medical School, University of

Newcastle

J Pooley

Correspondence to:

Dr Datta.

email:h.k.datta@

newcastle.ac.uk

Accepted for publication

9 December 1997

Table 1 Clinical features and mitochondrial DNA deletions in bone

\begin{tabular}{lllll}
\hline Patient & Age/sex & Pathology & Procedure & $\begin{array}{l}\text { mtDNA } \\
\text { deletion }\end{array}$ \\
\hline 1 & $31 / \mathrm{M}$ & Osteoarthritis after RTA & Left THR & - \\
2 & $73 / \mathrm{F}$ & Osteoporosis/rheumatoid arthritis & Right TKR & + \\
3 & $74 / \mathrm{F}$ & Osteoporosis/rheumatoid arthritis & Left TKR & - \\
4 & $74 / \mathrm{M}$ & Osteoporosis/rheumatoid arthritis & Left TKR & + \\
5 & $85 / \mathrm{M}$ & Osteoporosis & BHR & + \\
6 & $82 / \mathrm{F}$ & Osteoporosis & Right THR & ND \\
7 & $49 / \mathrm{F}$ & Acromegaly/osteoporosis & Right TKR & - \\
8 & $52 / \mathrm{F}$ & Osteoporosis & Right THR & - \\
9 & $56 / \mathrm{F}$ & Rotator cuff tear & AA & - \\
10 & $58 / \mathrm{F}$ & Rotator cuff tear & AA & - \\
\hline
\end{tabular}

+ , Deletion in the $8.4 \mathrm{~kb}$ mtDNA fragment amplified using PCR; -, no deletion.

RTA, road traffic accident; THR, total hip replacement; TKR, total knee replacement; BHR, bilateral hip replacement; AA, anterior acromioplasty; ND, PCR amplification could not be done.

ity reaches a peak between declines. The decline accelerates in both sexes between the fifth and sixth decades. The accelerated bone loss seen following the menopause is often attributed to oestrogen deficiency. However, although men do not experience an accelerated bone loss comparable with that seen in women, they do have an age related decrease in bone density. Therefore, the aging process itself is associated with bone loss leading to a diminution in bone mineral density, increased microarchitectural deterioration, and increased susceptibility to osteoporosis.

The time dependent accumulation of somatic DNA mutations may produce inefficient cell function, leading to deleterious degenerative tissue changes characteristic of aging. ${ }^{12}$ Age related, somatic mitochondrial DNA (mtDNA) mutation occurs and tends to accumulate with time owing to the lack of a mtDNA repair system. ${ }^{3}$ Some of these somatic mutations in the mitochondrial genome result in a variety of clinical manifestations including neurological disorders, cardiomyopathy, liver disease, and renal dysfunction. Symptoms of most of the mtDNA related diseases do not manifest until late in life and rapidly progress thereafter. ${ }^{4}$ In view of these facts and the fact that osteoporosis is primarily a disease of the elderly, the search for a link between mtDNA mutations and age related, accelerated bone loss leading to osteoporosis needs to be investigated. Late onset of bone loss leading to osteoporosis could be explained by the cumulative effect of somatic mtDNA mutations because there is a rapid rise in these mutations late in life. In the present study, we carried out selective amplification of mtDNA to detect any specific "common deletion" in the bone and blood of selected individuals who had undergone orthopaedic surgery.

\section{Materials and methods}

SUBJECT SELECTION

Bone and blood samples were obtained from six subjects who had undergone orthopaedic surgery (table 1): a 31 year old man with hip arthroplasty (patient 1) and a history of osteoarthritis secondary to a road traffic accident; three subjects with total left knee replacement, two of whom were women aged 73 and 74 years (patients 2 and 3, respectively), the third was a 74 year old man (patient 4); two elderly subjects, a man aged 85 years (patient 5) and a woman aged 82 years (patient 6 ), with hip arthroplasty following a non-traumatic fracture of the femoral neck of the femur. A further four bone biopsies but no blood samples were obtained from patients aged 49, 52, 56, and 58 years (patients 7, 8, 9, and 10, respectively). None of the patients had been treated with cytotoxic drugs and there was no history of alcohol abuse. Biochemical profiles including liver function tests and blood counts were within reference range in all patients.

\section{DNA ISOLATION}

Human bone from orthopaedic surgery was curetted into small pieces, placed in $1.5 \mathrm{ml}$ 


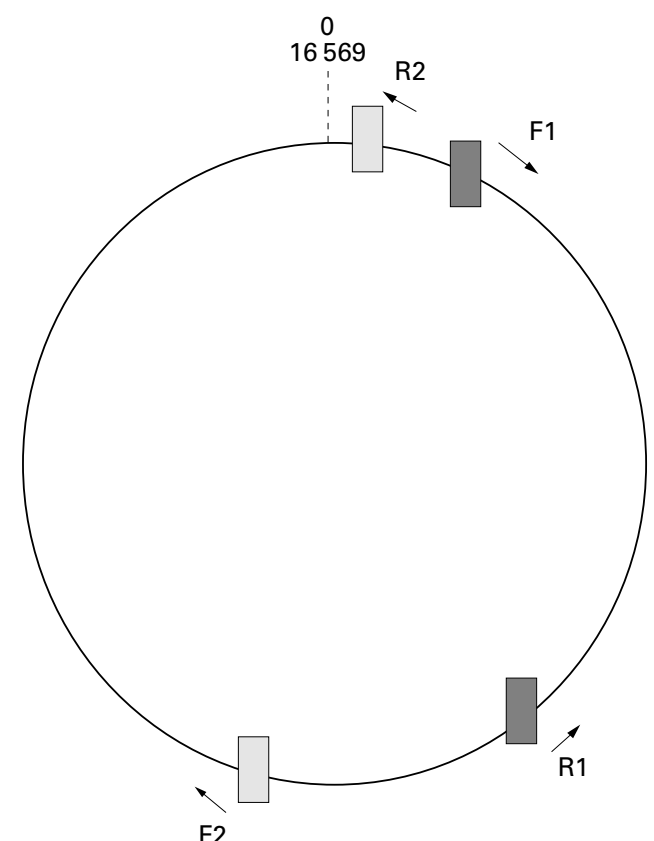

Figure 1 Schematic diagram of the region of mitochondrial DNA amplified. The arrows indicate the site of primers, darker boxes denote the region of the primers used for amplifying the $5 \mathrm{~kb}$ fragment and lighter boxes denote the site of primers used for the amplification of the $8.4 \mathrm{~kb}$ fragment. The vertical line designates the origin of mitochondrial replication.

ESP solution $(0.5 \mathrm{mM}$ ethylenediamine tetra acetic acid, $\mathrm{pH} 9,0.019 \mathrm{mg} / \mathrm{ml}$ sodium dodecyl sulphate, $1 \mathrm{mg} / \mathrm{ml}$ proteinase $\mathrm{K}$ ) and incubated at $37^{\circ} \mathrm{C}$ for three days. Genomic DNA was isolated from the bone extract by adding $0.5 \mathrm{ml} \mathrm{phenol/chloroform} \mathrm{to} 0.5 \mathrm{ml}$ bone ESP extract and shaking for 10 seconds; the aqueous phase was recovered by centrifugation. To further purify DNA samples, DNA Wizard microcolumns (Promega, Southampton, UK) were used. DNA from blood leucocytes was isolated using a Nucleon kit (Scotlab, Paisley, UK).

DETECTION OF mtDNA DELETIONS USING PCR Polymerase chain reaction (PCR) amplification was carried out to detect $5 \mathrm{~kb}$ and $8.4 \mathrm{~kb}$ products of mtDNA isolated from the blood and bone of the six subjects. The analysis was

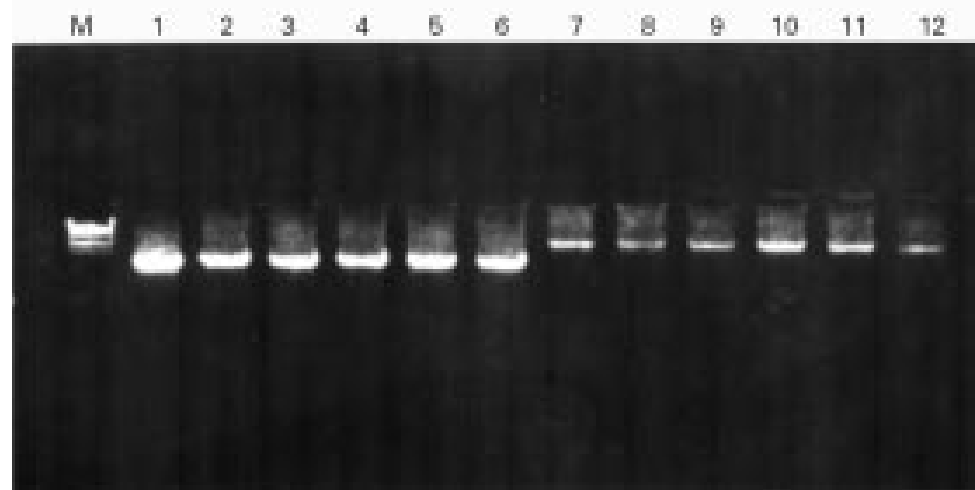

Figure 2 Amplification of blood DNA from six individuals of different ages. The amplification of the $5 \mathrm{~kb}$ (lanes 1-6) and $8.4 \mathrm{~kb}$ (lanes 7-12) fragments of mtDNA using primers $F 1$ and $R 1$ and $F 2$ and $R 2$, respectively, showed all amplifications were successful and that there were no deletions. $M$ represents size markers. carried out in $50 \mu 1$ of total reaction volume comprising: $2.5 \mu \mathrm{l}$ primers $(10 \mathrm{mM}), 2 \mu \mathrm{l}$ dNTPs $(5 \mathrm{mM}), 1 \mu \mathrm{l}$ of Long Expand polymerase (Sigma, Poole, Dorset, UK), and $1 \mu \mathrm{l}$ of DNA (10 ng); reactions were covered with $50 \mu$ paraffin oil (Sigma). The sites of the mitochondrial genome amplified are shown in the schematic diagram (fig 1). The primers used for the amplification of the $5 \mathrm{~kb}$ fragment covered the Cambridge sequence from 1180 to 6190 ; the forward primer $(\mathrm{F} 1)$, covering the complimentary site 1161 to 1180 , was: 5'-AACTCAAAGGACCTGGCGGT-3' and the reverse primer (R1), covering the site 6171 to 6190, was: 5'CGGGGAAACGCCATATCGGG-3'. Amplification of the $8.4 \mathrm{~kb}$ fragment, covering the stretch of mitochondria from 8550 to 400, was carried out using the forward primer (F2): 5'-ACGAAAATCTGTTCGCTTCA-3' and the reverse primer (R2): 5'AAATTTGAAATCTGGTTAGG-3'. Reactions were carried out in a Perkin Elmer Cetus thermal cycler; conditions for the amplification of the $5 \mathrm{~kb}$ fragment were as follows: denaturation, 15 seconds at $94^{\circ} \mathrm{C}$; annealing, 80 seconds at $65^{\circ} \mathrm{C}$; and extension, four minutes at $68^{\circ} \mathrm{C}$. Conditions for the amplification of the $8.4 \mathrm{~kb}$ fragment were as follows: denaturation, 15 seconds at $94^{\circ} \mathrm{C}$; annealing, 30 seconds at $60^{\circ} \mathrm{C}$; and extension, six minutes at $68^{\circ} \mathrm{C}$. PCR products were separated by electrophoresis on $1 \%$ agarose gels containing ethidium bromide and were detected and photographed under UV light.

\section{Results}

No mtDNA deletions were detected in the leucocytes of any of the six subjects. However, we found almost complete loss of normal mtDNA from the bone of a male subject with severe osteoporosis leading to bilateral hip fracture. Of the remaining patients, an elderly man exhibited similarly large deletions and a smaller deletion was observed in the bone mtDNA from an elderly woman, thus providing evidence for bone related, somatic deletions in bone mtDNA. Interestingly, none of the patients younger than 60 years showed any evidence of mtDNA deletions (table 1).

PCR amplification generated the expected $5 \mathrm{~kb}$ and $8.4 \mathrm{~kb}$ fragments with mtDNA isolated from blood samples of the six patients using primers $\mathrm{F} 1, \mathrm{R} 1$ and $\mathrm{F} 2, \mathrm{R} 2$, respectively (fig 2). Analysis of bone mtDNA from five subjects revealed that although the $5 \mathrm{~kb}$ amplification product showed no evidence of any deletion (fig 3A) three patients had deletions in the $8.4 \mathrm{~kb}$ fragment (fig 3B). Of these three patients, two with similar sized deletions were male and the third, a female, had a smaller deletion. Isolation of poor quality DNA from the bone of one of the patients hindered PCR amplification.

The youngest subject, a 31 year old man with osteoarthritis resulting from a road traffic accident who underwent left hip arthroplasty, and a 74 year old woman showed no evidence of mtDNA deletions (fig 3B). Four other bone biopsies from hip and knee from younger 
A

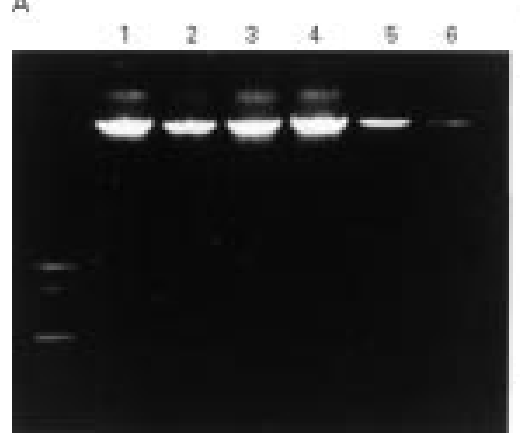

a

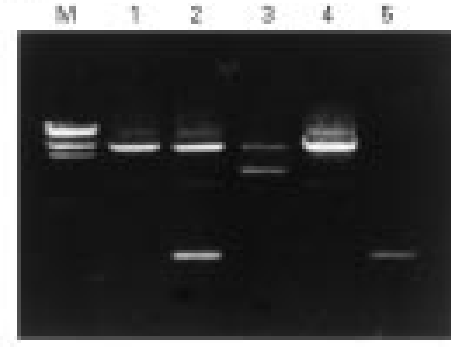

Figure 3 Amplification of bone DNA obtained from five individuals of different ages who had undergone orthopaedic surgery. (A) Amplification of the $5 \mathrm{~kb}$ fragment of bone $m t D N A$ showed that there were no deletions. (B) Amplification of the $8.4 \mathrm{~kb} m \mathrm{mDNA}$ fragment showed that three of the five samples possessed deletions (lanes 2, 3, and 5). Two of the deletions were quite similar in size (lanes 2 and 5) and were present in an osteoporotic 85 year old man and a 73 year old man, respectively. A third smaller deletion producing a larger fragment was found in a 75 year old woman (lane 3). $M$ represents size markers. patients with age ranges of 45 to 59 years showed no mtDNA deletions (data not shown). The most severe degree of $\mathrm{mtDNA}$ deletion was seen in the oldest subject, an 85 year old man who had bilateral hip arthroplasty following non-traumatic fractures (fig 3B). In this subject, the $8.4 \mathrm{~kb}$ fragment was undetectable, suggesting that almost all the mtDNA possessed the deletion. Interestingly, another male patient (74 years old) who had undergone left total knee arthroplasty, produced an almost identical deletion upon amplification of the $8.4 \mathrm{~kb}$ fragment (fig 3B). However, it is evident that over $50 \%$ of the mitochondria in this individual showed no deletion. Finally, a third deletion was found in a 73 year old female subject; the size of the deletion was small, hence the DNA fragment amplified is large, and about half of the total mtDNA was affected (fig 3B).

\section{Discussion}

This is the first attempt at detecting age associated mtDNA deletions in bone. Recent studies have reported a wide variation in the levels of mtDNA deletions in different tissues of elderly subjects. ${ }^{1-3}$ There appears to be two groups of tissues in terms of the extent of deletion: one with a relatively high deletion level and the other with a relatively low deletion level. The group of tissues with high deletion levels tends to be metabolically more active and includes brain, cardiac muscle, and skeletal muscle. The tissues with a lower deletion level tend to have a lower metabolic rate and include skin, liver, and spleen. A plausible explanation for this observation is thought to be that higher metabolic rates result in an increase in oxidative phosphorylation within mitochondria leading to production of damaging oxygen species such as superoxide anions, singlet oxygen, and hydroxyl radicals as a side-reaction of electron transport. The production of free radicals within mitochondria induces damage to mtDNA; this damage could include a single base change that increases free radical production, eventually leading to severe deletions.

The fact that tissues with high mitotic rates tend to accumulate low mtDNA deletions, even when they have high metabolic rates, might be explained by the recent hypothesis that apoptosis might be a selective mechanism for the elimination of superoxide producing mitochondria. ${ }^{56}$ We believe that apoptosis of cells bearing deletions of mtDNA may occur more efficiently in cells with high mitotic activity. According to this hypothesis, increased production of free radicals induces the release of a special "cell suicide protein" or "apoptosis inducing factor", normally sequestered in the mitochondrial intermembrane, into the cytosol.

Although the overall metabolic rate of bone is low, osteoblasts are actively metabolising cells with a high mitotic index. Therefore, in younger adults with high metabolic and mitotic rates, we expect to see lower levels of bone mtDNA deletion because such deletions would be eliminated by apoptosis, as indeed is the case. We contend that in the elderly, although the metabolic rate of osteoblasts may remain the same or decline marginally, there tends to be a more dramatic decline in the mitotic rate with the following consequences. First, a low mitotic rate means that cells possessing deletions cannot be eliminated as quickly as cells with high mitotic rates; hence there will be an increase in deletions with aging per se as well as in other conditions producing low osteoblast turnover, such as prolonged hypogonadism. The result of the age related accumulation of mtDNA deletions is accelerated by bone loss by normal osteoblast apoptosis. Therefore, we speculate that for those at risk of developing accelerated bone loss, early institution of treatment would be expected to produce a better response; delay in treatment would allow the cells with mtDNA deletions to accumulate, causing a decline in the osteoblast population.

The accumulation of mtDNA mutations in aging human tissues has been extensively investigated. ${ }^{4}$ However, other causes of mtDNA mutations have received less attention. Recent studies have shown mtDNA abnormalities in human neoplastic tissues, which may contribute to carcinogenesis, such as in renal cell carcinoma and breast malignancy. ${ }^{7}$ Increased expression of cytochrome $\mathrm{C}$ oxidase subunit 2 (COII) was found in primary malignant breast tissue. ${ }^{8}$ The content of intracellular mitochondrial DNA is decreased by 1-methyl-4-phenylpyridinium ion (MPP+), an oxidative metabolite of 1-methyl4-phenyl-1, 2, 3, 6-tetrahydropyridine (MPTP) that induces Parkinson's disease-like symptoms. ${ }^{9}$ The inhibition of the mtDNA replication is also produced by 3'-azido-3'deoxythymidine and other dideoxynucleoside analogues that inhibit human immunodeficiency virus 1 (HIV-1) replication. ${ }^{10}$ However, the involvement of xenobiotics in initiating mtDNA mutations has not been documented.

Finally, it is interesting that mtDNA deletions accumulate in tissues that show age related dysfunction, such as cardiac muscle, brain, and according to the present study, bone. Further work is required to define precisely the role of mtDNA mutations in the bone and their association with bone loss and the development of osteoporotic fractures. 
We are grateful to Theresa Wardell for technical assistance. This work was supported by the National Osteoporosis Society and The Wishbone Trust (HKD).

1 Holt IJ, Harding AE, Morgan-Hughes JA. Deletions of muscle mitochondrial DNA in patients with mitochondrial muscle mitochondrial DNA in patients
myopathies. Nature 1988;331:717-19.

myopathies. Nature 1988;331:717-19.
2 Cortopassi GA, Shibata D, Soong NW, et al. A pattern of accumulation of a somatic deletion of mitochondrial DNA in aging tissues. Proc Natl Acad Sci USA 1992;89: $7370-4$.

3 Simonetti S, Chen X, DiMauro S, et al. Accumulations of deletion of mitochondrial DNA during normal aging: analysis by quantitative PCR. Biochem Biophys Acto 1992;1180:113-22.

4 Wallace, DC. Mitochondrial DNA: a paradigm for aging and degenerative disease? Science 1992;256:628-32.

5 Petit PX, Susin SA, Zamzami N, et al. Mitochondria and programmed cell death: back to the future. FEBS Lett 1996;396:7-13.
6 Skulachev VP. Why are mitochondria involved in apoptosis? Permeability transition pores and apoptosis as selective mechanisms to eliminate superoxide producing mitochondria and cells. FEBS Lett 1996;397:7-10.

7 Horton TM, Petros JA, Heddi A, et al. Novel mitochondrial DNA deletion found in a renal cell carcinoma. Genes Chromosomes Cancer 1996;15:95-101.

8 Sharp MGF, Adams SM, Walker RA, et al. Differential expression of the mitochondrial gene cytochrome oxidase II in benign and malignant breast tissues. $f$ Pathol 1992;168:163-8.

9 Miyako K, Kai Y, Irie T, et al. The content of intracellular mitochondrial DNA is decreased by 1-methyl-4phenylpyridinium ion $(\mathrm{MPP}+)$. F Biol Chem 1997;272: 9605-8

10 Simpson MV, Chin CD, Keilbaugh SA, et al. Studies on the inhibition of the mitochondrial DNA replication by 3'-azido-3'-deoxythymidine and other dideoxynucleoside analogues which inhibit HIV-1 replication. Biochem Pharmacol 1989;38:1033-8. 